\title{
A Modified Halpern-Type Iterative Method of a System of Equilibrium Problems and a Fixed Point for a Totally Quasi- $\phi$-Asymptotically Nonexpansive Mapping in a Banach Space
}

\author{
Preedaporn Kanjanasamranwong, ${ }^{1}$ Poom Kumam, ${ }^{2}$ \\ and Siwaporn Saewan ${ }^{1}$ \\ ${ }^{1}$ Department of Mathematics and Statistics, Faculty of Science, Thaksin University (TSU), Pa Phayom, \\ Phatthalung 93110, Thailand \\ ${ }^{2}$ Department of Mathematics, Faculty of Science, King Mongkut's University of Technology Thonburi \\ (KMUTT), Bangmod, Bangkok 10140, Thailand
}

Correspondence should be addressed to Poom Kumam, poom.kum@kmutt.ac.th and Siwaporn Saewan, si_wa_pon@hotmail.com

Received 17 February 2012; Accepted 28 April 2012

Academic Editor: Yeol Je Cho

Copyright (C) 2012 Preedaporn Kanjanasamranwong et al. This is an open access article distributed under the Creative Commons Attribution License, which permits unrestricted use, distribution, and reproduction in any medium, provided the original work is properly cited.

The purpose of this paper is to introduce the modified Halpern-type iterative method by the generalized $f$-projection operator for finding a common solution of fixed-point problem of a totally quasi- $\phi$-asymptotically nonexpansive mapping and a system of equilibrium problems in a uniform smooth and strictly convex Banach space with the Kadec-Klee property. Consequently, we prove the strong convergence for a common solution of above two sets. Our result presented in this paper generalize and improve the result of Chang et al., (2012), and some others.

\section{Introduction}

In 1953, Mann [1] introduced the following iteration process which is now known as Mann's iteration:

$$
x_{n+1}=\alpha_{n} x_{n}+\left(1-\alpha_{n}\right) T x_{n}
$$

where $T$ is nonexpansive, the initial guess element $x_{1} \in C$ is arbitrary, and $\left\{\alpha_{n}\right\}$ is a sequence in $[0,1]$. Mann iteration has been extensively investigated for nonexpansive mappings. In an 
infinite-dimensional Hilbert space, Mann iteration can conclude only weak convergence (see $[2,3])$.

Later, in 1967, Halpern [4] considered the following algorithm:

$$
x_{1} \in C, \quad x_{n+1}=\alpha_{n} x_{1}+\left(1-\alpha_{n}\right) T x_{n}, \quad \forall n \geq 0,
$$

where $T$ is nonexpansive. He proved the strong convergence theorem of $\left\{x_{n}\right\}$ to a fixed point of $T$ under some control condition $\left\{\alpha_{n}\right\}$. Many authors improved and studied the result of Halpern [4] such as Qin et al. [5], Wang et al. [6], and reference therein.

In 2008-2009, Takahashi and Zembayashi [7, 8] studied the problem of finding a common element of the set of fixed points of a nonexpansive mapping and the set of solutions of an equilibrium problem in the framework of the Banach spaces.

On the other hand, Li et al. [9] introduced the following hybrid iterative scheme for approximation fixed points of relatively nonexpansive mapping using the generalized $f$ projection operator in a uniformly smooth real Banach space which is also uniformly convex. They obtained strong convergence theorem for finding an element in the fixed point set of $T$.

Recently, Ofoedu and Shehu [10] extended algorithm of Li et al. [9] to prove a strong convergence theorem for a common solution of a system of equilibrium problem and the set of common fixed points of a pair of relatively quasi-nonexpansive mappings in the Banach spaces by using generalized $f$-projection operator. Chang et al. [11] extended and improved Qin and Su [12] to obtain a strong convergence theorem for finding a common element of the set of solutions for a generalized equilibrium problem, the set of solutions for a variational inequality problem, and the set of common fixed points for a pair of relatively nonexpansive mappings in a Banach space.

Very recently, Chang et al. [13] extended the results of Qin et al. [5] and Wang et al. [6] to consider a modification to the Halpern-type iteration algorithm for a total quasi- $\phi$-asymptotically nonexpansive mapping to have the strong convergence under a limit condition only in the framework of Banach spaces.

The purpose of this paper is to be motivated and inspired by the works mentioned above, we introduce a modified Halpern-type iterative method by using the new hybrid projection algorithm of the generalized $f$-projection operator for solving the common solution of fixed point for totally quasi- $\phi$-asymptoically nonexpansive mappings and the system of equilibrium problems in a uniformly smooth and strictly convex Banach space with the Kadec-Klee property. The results presented in this paper improve and extend the corresponding ones announced by many others.

\section{Preliminaries and Definitions}

Let $E$ be a real Banach space with dual $E^{*}$, and let $C$ be a nonempty closed and convex subset of $E$. Let $\left\{\theta_{i}\right\}_{i \in \Gamma}: C \times C \rightarrow \mathbb{R}$ be a bifunction, where $\Gamma$ is an arbitrary index set. The system of equilibrium problems is to find $x \in C$ such that

$$
\theta_{i}(x, y) \geq 0, \quad i \in \Gamma, \forall y \in C
$$


If $\Gamma$ is a singleton, then problem (2.1) reduces to the equilibrium problem, which is to find $x \in C$ such that

$$
\theta(x, y) \geq 0, \quad \forall y \in C
$$

A mapping $T$ from $C$ into itself is said to be nonexpansive if

$$
\|T x-T y\| \leq\|x-y\|, \quad \forall x, y \in C
$$

$T$ is said to be asymptotically nonexpansive if there exists a sequence $\left\{k_{n}\right\} \subset[1, \infty)$ with $k_{n} \rightarrow 1$ as $n \rightarrow \infty$ such that

$$
\left\|T^{n} x-T^{n} y\right\| \leq k_{n}\|x-y\|, \quad \forall x, y \in C .
$$

$T$ is said to be total asymptotically nonexpansive if there exist nonnegative real sequences $v_{n}, \mu_{n}$ with $v_{n} \rightarrow 0, \mu_{n} \rightarrow 0$ as $n \rightarrow \infty$ and a strictly increasing continuous function $\varphi: \mathbb{R}^{+} \rightarrow \mathbb{R}^{+}$ with $\varphi(0)=0$ such that

$$
\left\|T^{n} x-T^{n} y\right\| \leq\|x-y\|+v_{n} \psi(\|x-y\|)+\mu_{n}, \quad \forall x, y \in C, \forall n \geq 1 .
$$

A point $x \in C$ is a fixed point of $T$ provided $T x=x$. Denote by $F(T)$ the fixed point set of $T$; that is, $F(T)=\{x \in C: T x=x\}$. A point $p$ in $C$ is called an asymptotic fixed point of $T$ if $C$ contains a sequence $\left\{x_{n}\right\}$ which converges weakly to $p$ such that $\lim _{n \rightarrow \infty}\left\|x_{n}-T x_{n}\right\|=0$. The asymptotic fixed point set of $T$ is denoted by $\widehat{F}(T)$.

The normalized duality mapping $J: E \rightarrow 2^{E^{*}}$ is defined by $J(x)=\left\{x^{*} \in E^{*}:\left\langle x, x^{*}\right\rangle=\right.$ $\left.\|x\|^{2},\left\|x^{*}\right\|=\|x\|\right\}$. If $E$ is a Hilbert space, then $J=I$, where $I$ is the identity mapping. Consider the functional defined by

$$
\phi(x, y)=\|x\|^{2}-2\langle x, J y\rangle+\|y\|^{2},
$$

where $J$ is the normalized duality mapping and $\langle\cdot, \cdot\rangle$ denote the duality pairing of $E$ and $E^{*}$. If $E$ is a Hilbert space, then $\phi(y, x)=\|y-x\|^{2}$. It is obvious from the definition of $\phi$ that

$$
(\|y\|-\|x\|)^{2} \leq \phi(y, x) \leq(\|y\|+\|x\|)^{2}, \quad \forall x, y \in E .
$$

A mapping $T$ from $C$ into itself is said to be $\phi$-nonexpansive $[14,15]$ if

$$
\phi(T x, T y) \leq \phi(x, y), \quad \forall x, y \in C .
$$

$T$ is said to be quasi- $\phi$-nonexpansive $[14,15]$ if $F(T) \neq \emptyset$ and

$$
\phi(p, T x) \leq \phi(p, x), \quad \forall x \in C, p \in F(T) .
$$


$T$ is said to be asymptotically $\phi$-nonexpansive [15] if there exists a sequence $\left\{k_{n}\right\} \subset[0, \infty)$ with $k_{n} \rightarrow 1$ as $n \rightarrow \infty$ such that

$$
\phi\left(T^{n} x, T^{n} y\right) \leq k_{n} \phi(x, y), \quad \forall x, y \in C .
$$

$T$ is said to be quasi- $\phi$-asymptotically nonexpansive [15] if $F(T) \neq \emptyset$ and there exists a sequence $\left\{k_{n}\right\} \subset[0, \infty)$ with $k_{n} \rightarrow 1$ as $n \rightarrow \infty$ such that

$$
\phi\left(p, T^{n} x\right) \leq k_{n} \phi(p, x), \quad \forall x \in C, p \in F(T), \forall n \geq 1
$$

$T$ is said to be totally quasi- $\phi$-asymptotically nonexpansive, if $F(T) \neq \emptyset$ and there exist nonnegative real sequences $v_{n}, \mu_{n}$ with $v_{n} \rightarrow 0, \mu_{n} \rightarrow 0$ as $n \rightarrow \infty$ and a strictly increasing continuous function $\varphi: \mathbb{R}^{+} \rightarrow \mathbb{R}^{+}$with $\varphi(0)=0$ such that

$$
\phi\left(p, T^{n} x\right) \leq \phi(p, x)+v_{n} \varphi(\phi(p, x))+\mu_{n}, \quad \forall n \geq 1, \forall x \in C, p \in F(T) .
$$

A mapping $T$ from $C$ into itself is said to be closed if for any sequence $\left\{x_{n}\right\} \subset C$ such that $\lim _{n \rightarrow \infty} x_{n}=x_{0}$ and $\lim _{n \rightarrow \infty} T x_{n}=y_{0}$, then $T x_{0}=y_{0}$.

Alber [16] introduced the generalized projection $\Pi_{C}: E \rightarrow C$ is a map that assigns to an arbitrary point $x \in E$ the minimum point of the functional $\phi(x, y)$; that is, $\Pi_{C} x=\bar{x}$, where $\bar{x}$ is the solution of the minimization problem:

$$
\phi(\bar{x}, x)=\inf _{y \in C} \phi(y, x)
$$

The existence and uniqueness of the operator $\Pi_{C}$ follows from the properties of the functional $\phi(y, x)$ and the strict monotonicity of the mapping $J$ (see, e.g., [16-20]). If $E$ is a Hilbert space, then $\phi(x, y)=\|x-y\|^{2}$ and $\Pi_{C}$ becomes the metric projection $P_{C}: H \rightarrow C$. If $C$ is a nonempty, closed, and convex subset of a Hilbert space $H$, then $P_{C}$ is nonexpansive. This fact actually characterizes Hilbert spaces, and consequently, it is not available in more general Banach spaces. Later, $\mathrm{Wu}$ and Huang [21] introduced a new generalized $f$-projection operator in the Banach space. They extended the definition of the generalized projection operators and proved some properties of the generalized $f$-projection operator. Next, we recall the concept of the generalized $f$-projection operator. Let $G: C \times E^{*} \rightarrow \mathbb{R} \cup\{+\infty\}$ be a functional defined by

$$
G(y, \varpi)=\|y\|^{2}-2\langle y, \varpi\rangle+\|\varpi\|^{2}+2 \rho f(y),
$$

where $y \in C, \varpi \in E^{*}, \rho$ is positive number, and $f: C \rightarrow \mathbb{R} \cup\{+\infty\}$ is proper, convex, and lower semicontinuous. From the definition of $G, \mathrm{Wu}$ and Huang [21] proved the following properties:

(1) $G(y, \varpi)$ is convex and continuous with respect to $\varpi$ when $y$ is fixed;

(2) $G(y, \varpi)$ is convex and lower semicontinuous with respect to $y$ when $\varpi$ is fixed. 
Definition 2.1. Let $E$ be a real Banach space with its dual $E^{*}$. Let $C$ be a nonempty, closed, and convex subset of $E$. We say that $\pi_{C}^{f}: E^{*} \rightarrow 2^{C}$ is a generalized $f$-projection operator if

$$
\pi_{C}^{f} \varpi=\left\{u \in C: G(u, \varpi)=\inf _{y \in C} G(y, \varpi), \forall \varpi \in E^{*}\right\}
$$

A Banach space $E$ with norm $\|\cdot\|$ is called strictly convex if $\|(x+y) / 2\|<1$ for all $x, y \in E$ with $\|x\|=\|y\|=1$ and $x \neq y$. Let $U=\{x \in E:\|x\|=1\}$ be the unit sphere of $E$. A Banach space $E$ is called smooth if the $\operatorname{limit}_{t \rightarrow 0} \lim _{t \rightarrow 0}((\|x+t y\|-\|x\|) / t)$ exists for each $x, y \in U$. It is also called uniformly smooth if the limit exists uniformly for all $x, y \in U$. The modulus of smoothness of $E$ is the function $\rho_{E}:[0, \infty) \rightarrow[0, \infty)$ defined by $\rho_{E}(t)=\sup \{(\|x+y\|+\|x-y\|) / 2-1$ : $\|x\|=1,\|y\| \leq t\}$. The modulus of convexity of $E$ (see [22]) is the function $\delta_{E}:[0,2] \rightarrow[0,1]$ defined by $\delta_{E}(\varepsilon)=\inf \{1-\|(x+y) / 2\|: x, y \in E,\|x\|=\|y\|=1,\|x-y\| \geq \varepsilon\}$. In this paper we denote the strong convergence and weak convergence of a sequence $\left\{x_{n}\right\}$ by $x_{n} \rightarrow x$ and $x_{n} \rightarrow x$, respectively.

Remark 2.2. The basic properties of $E, E^{*}, J$, and $J^{-1}$ (see [18]) are as follows.

(i) If $E$ is an arbitrary Banach space, then $J$ is monotone and bounded.

(ii) If $E$ is a strictly convex, then $J$ is strictly monotone.

(iii) If $E$ is a smooth, then $J$ is single valued and semicontinuous.

(iv) If $E$ is uniformly smooth, then $J$ is uniformly norm-to-norm continuous on each bounded subset of $E$.

(v) If $E$ is reflexive smooth and strictly convex, then the normalized duality mapping $J$ is single valued, one-to-one, and onto.

(vi) If $E$ is a reflexive strictly convex and smooth Banach space and $J$ is the duality mapping from $E$ into $E^{*}$, then $J^{-1}$ is also single valued, bijective, and is also the duality mapping from $E^{*}$ into $E$, and thus $J J^{-1}=I_{E^{*}}$ and $J^{-1} J=I_{E}$.

(vii) If $E$ is uniformly smooth, then $E$ is smooth and reflexive.

(viii) $E$ is uniformly smooth if and only if $E^{*}$ is uniformly convex.

(ix) If $E$ is a reflexive and strictly convex Banach space, then $J^{-1}$ is norm-weak*continuous.

Remark 2.3. If $E$ is a reflexive, strictly convex, and smooth Banach space, then $\phi(x, y)=0$, if and only if $x=y$. It is sufficient to show that if $\phi(x, y)=0$ then $x=y$. From (2.6), we have $\|x\|=\|y\|$. This implies that $\langle x, J y\rangle=\|x\|^{2}=\|J y\|^{2}$. From the definition of $J$, one has $J x=J y$. Therefore, we have $x=y$ (see $[18,20,23]$ for more details).

Recall that a Banach space $E$ has the Kadec-Klee property [18, 20, 24], if for any sequence $\left\{x_{n}\right\} \subset E$ and $x \in E$ with $x_{n} \rightarrow x$ and $\left\|x_{n}\right\| \rightarrow\|x\|$, then $\left\|x_{n}-x\right\| \rightarrow 0$ as $n \rightarrow \infty$. It is well known that if $E$ is a uniformly convex Banach space, then $E$ has the Kadec-Klee property.

We also need the following lemmas for the proof of our main results.

Lemma 2.4 (see Change et al. [25]). Let $C$ be a nonempty, closed, and convex subset of a uniformly smooth and strictly convex Banach space $E$ with the Kadec-Klee property. Let $T: C \rightarrow C$ be a closed 
and total quasi- $\phi$-asymptotically nonexpansive mapping with nonnegative real sequence $\nu_{n}$ and $\mu_{n}$ with $v_{n} \rightarrow 0, \mu_{n} \rightarrow 0$ as $n \rightarrow \infty$ and a strictly increasing continuous function $\zeta: \mathbb{R}^{+} \rightarrow \mathbb{R}^{+}$with $\zeta(0)=0$. If $\mu_{1}=0$, then the fixed point set $F(T)$ is a closed convex subset of $C$.

Lemma 2.5 (see Wu and Hung [21]). Let E be a real reflexive Banach space with its dual $E^{*}$ and C a nonempty, closed, and convex subset of $E$. The following statement hold:

(1) $\pi_{C}^{f} \varpi$ is a nonempty, closed and convex subset of $C$ for all $\varpi \in E^{*}$;

(2) if $E$ is smooth, then for all $\varpi \in E^{*}, x \in \pi_{C}^{f} \varpi$ if and only if

$$
\langle x-y, \varpi-J x\rangle+\rho f(y)-\rho f(x) \geq 0, \quad \forall y \in C
$$

(3) if $E$ is strictly convex and $f: C \rightarrow \mathbb{R} \cup\{+\infty\}$ is positive homogeneous (i.e., $f(t x)=t f(x)$ for all $t>0$ such that $t x \in C$ where $x \in C)$, then $\pi_{C}^{f} \varpi$ is single-valued mapping.

Lemma 2.6 (see Fan et al. [26]). Let E be a real reflexive Banach space with its dual $E^{*}$ and $C$ be a nonempty, closed and convex subset of $E$. If $E$ is strictly convex, then $\pi_{C}^{f} \varpi$ is single valued.

Recall that $J$ is single-valued mapping when $E$ is a smooth Banach space. There exists a unique element $\varpi \in E^{*}$ such that $\varpi=J x$ where $x \in E$. This substitution in (2.14) gives

$$
G(y, J x)=\|y\|^{2}-2\langle y, J x\rangle+\|x\|^{2}+2 \rho f(y) .
$$
[9]).

Now we consider the second generalized $f$ projection operator in Banach space (see

Definition 2.7. Let $E$ be a real smooth Banach space, and let $C$ be a nonempty, closed, and convex subset of $E$. We say that $\Pi_{C}^{f}: E \rightarrow 2^{C}$ is generalized $f$-projection operator if

$$
\Pi_{C}^{f} x=\left\{u \in C: G(u, J x)=\inf _{y \in C} G(y, J x), \forall x \in E\right\} .
$$

Lemma 2.8 (see Deimling [27]). Let $E$ be a Banach space, and let $f: E \rightarrow \mathbb{R} \cup\{+\infty\}$ be a lower semicontinuous convex function. Then there exist $x^{*} \in E^{*}$ and $\alpha \in \mathbb{R}$ such that

$$
f(x) \geq\left\langle x, x^{*}\right\rangle+\alpha, \quad \forall x \in E .
$$

Lemma 2.9 (see Li et al. [9]). Let $E$ be a reflexive smooth Banach space, and let $C$ be a nonempty, closed, and convex subset of $E$. The following statements hold:

(1) $\Pi_{C}^{f} x$ is nonempty, closed and convex subset of $C$ for all $x \in E$;

(2) for all $x \in E, \widehat{x} \in \Pi_{C}^{f} x$ if and only if

$$
\langle\widehat{x}-y, J x-J \widehat{x}\rangle+\rho f(y)-\rho f(\widehat{x}) \geq 0, \quad \forall y \in C ;
$$

(3) if $E$ is strictly convex, then $\Pi_{C}^{f}$ is single-valued mapping. 
Lemma 2.10 (see Li et al. [9]). Let $E$ be a real reflexive smooth Banach space, let $C$ be a nonempty, closed, and convex subset of $E, x \in E$, and let $\hat{x} \in \Pi_{C}^{f} x$. Then

$$
\phi(y, \widehat{x})+G(\widehat{x}, J x) \leq G(y, J x), \quad \forall y \in C
$$

Remark 2.11. Let $E$ be a uniformly convex and uniformly smooth Banach space and $f(x)=0$ for all $x \in E$, then Lemma 2.10 reduces to the property of the generalized projection operator considered by Alber [16].

If $f(y) \geq 0$ for all $y \in C$ and $f(0)=0$, then the definition of totally quasi- $\phi$ asymptotically nonexpansive $T$ is equivalent to if $F(T) \neq \emptyset$, and there exist nonnegative real sequences $v_{n}, \mu_{n}$ with $v_{n} \rightarrow 0, \mu_{n} \rightarrow 0$ as $n \rightarrow \infty$ and a strictly increasing continuous function $\zeta: \mathbb{R}^{+} \rightarrow \mathbb{R}^{+}$with $\zeta(0)=0$ such that

$$
G\left(p, T^{n} x\right) \leq G(p, x)+v_{n} \zeta G(p, x)+\mu_{n}, \quad \forall n \geq 1, \forall x \in C, \quad p \in F(T) .
$$

For solving the equilibrium problem for a bifunction $\theta: C \times C \rightarrow \mathbb{R}$, let us assume that $\theta$ satisfies the following conditions:

(A1) $\theta(x, x)=0$ for all $x \in C$;

(A2) $\theta$ is monotone; that is, $\theta(x, y)+\theta(y, x) \leq 0$ for all $x, y \in C$;

(A3) for each $x, y, z \in C$,

$$
\lim _{t \downarrow 0} \theta(t z+(1-t) x, y) \leq \theta(x, y)
$$

(A4) for each $x \in C, y \mapsto \theta(x, y)$ is convex and lower semicontinuous.

For example, let $A$ be a continuous and monotone operator of $C$ into $E^{*}$ and define

$$
\theta(x, y)=\langle A x, y-x\rangle, \quad \forall x, y \in C \text {. }
$$

Then, $\theta$ satisfies (A1)-(A4). The following result is in Blum and Oettli [28].

Lemma 2.12 (see Blum and Oettli [28]). Let $C$ be a closed convex subset of a smooth, strictly convex, and reflexive Banach space $E$, let $\theta$ be a bifunction from $C \times C$ to $\mathbb{R}$ satisfying (A1)-(A4), and let $r>0$ and $x \in E$. Then, there exists $z \in C$ such that

$$
\theta(z, y)+\frac{1}{r}\langle y-z, J z-J x\rangle \geq 0, \quad \forall y \in C
$$


Lemma 2.13 (see Takahashi and Zembayashi [8]). Let $C$ be a closed convex subset of a uniformly smooth, strictly convex, and reflexive Banach space $E$, and let $\theta$ be a bifunction from $C \times C$ to $\mathbb{R}$ satisfying conditions (A1)-(A4). For all $r>0$ and $x \in E$, define a mapping $T_{r}^{\theta}: E \rightarrow C$ as follows:

$$
T_{r}^{\theta} x=\left\{z \in C: \theta(z, y)+\frac{1}{r}\langle y-z, J z-J x\rangle \geq 0, \forall y \in C\right\} .
$$

Then the following hold:

(1) $T_{r}^{\theta}$ is single-valued;

(2) $T_{r}^{\theta}$ is a firmly nonexpansive-type mapping [29]; that is, for all $x, y \in E$,

$$
\left\langle T_{r}^{\theta} x-T_{r}^{\theta} y, J T_{r}^{\theta} x-J T_{r}^{\theta} y\right\rangle \leq\left\langle T_{r}^{\theta} x-T_{r}^{\theta} y, J x-J y\right\rangle
$$

(3) $F\left(T_{r}^{\theta}\right)=\operatorname{EP}(\theta)$;

(4) $\mathrm{EP}(\theta)$ is closed and convex.

Lemma 2.14 (see Takahashi and Zembayashi [8]). Let $C$ be a closed convex subset of a smooth, strictly convex, and reflexive Banach space $E$, let $\theta$ be a bifunction from $C \times C$ to $\mathbb{R}$ satisfying (A1)(A4), and let $r>0$. Then, for $x \in E$ and $q \in F\left(T_{r}^{\theta}\right)$,

$$
\phi\left(q, T_{r}^{\theta} x\right)+\phi\left(T_{r}^{\theta} x, x\right) \leq \phi(q, x)
$$

\section{Main Result}

Theorem 3.1. Let $C$ be a nonempty, closed, and convex subset of a uniformly smooth and strictly convex Banach space $E$ with the Kadec-Klee property. For each $j=1,2, \ldots, m$, let $\theta_{j}$ be a bifunction from $C \times C$ to $\mathbb{R}$ which satisfies conditions (A1)-(A4). Let $S: C \rightarrow C$ be a closed totally quasi- $\phi-$ asymptotically nonexpansive mappings with nonnegative real sequences $v_{n}, \mu_{n}$ with $v_{n} \rightarrow 0, \mu_{n} \rightarrow$ 0 as $n \rightarrow \infty$, and a strictly increasing continuous function $\psi: \mathbb{R}^{+} \rightarrow \mathbb{R}^{+}$with $\psi(0)=0$. Let $f: E \rightarrow \mathbb{R}$ be a convex and lower semicontinuous function with $C \subset$ int $(D(f))$ such that $f(x) \geq 0$ for all $x \in C$ and $f(0)=0$. Assume that $\mathcal{F}:=F(S) \cap\left(\cap_{j=1}^{m} \mathrm{EP}\left(\theta_{j}\right)\right) \neq \emptyset$. For an initial point $x_{1} \in E$ and $C_{1}=C$, one define the sequence $\left\{x_{n}\right\}$ by

$$
\begin{gathered}
u_{n}=T_{r_{m, n}}^{\theta_{m}} T_{r_{m-1, n}}^{\theta_{m-1}} T_{r_{m-2, n}}^{\theta_{m-2}} \cdots T_{r_{1, n}}^{\theta_{1}} x_{n}, \\
z_{n}=J^{-1}\left(\alpha_{n} J x_{1}+\left(1-\alpha_{n}\right) J S^{n} u_{n}\right), \\
C_{n+1}=\left\{v \in C_{n}: G\left(v, J z_{n}\right) \leq G\left(v, J u_{n}\right) \leq G\left(v, J x_{1}\right)+\left(1-\alpha_{n}\right) G\left(v, J x_{n}\right)+\zeta_{n}\right\}, \\
x_{n+1}=\prod_{C_{n+1}}^{f} x_{1}, \quad n \in \mathbb{N},
\end{gathered}
$$

where $\left\{\alpha_{n}\right\}$ is a sequence in $[0,1], \zeta_{n}=v_{n} \sup _{q \in \mathcal{F}} \psi\left(G\left(q, x_{n}\right)\right)+\mu_{n}$ and $\left\{r_{j, n}\right\} \subset[d, \infty)$ for some $d>0$. If $\lim _{n \rightarrow \infty} \alpha_{n}=0$, then $\left\{x_{n}\right\}$ converges strongly to $\Pi_{\mp}^{f} x_{0}$. 
Proof. We split the proof into four steps.

Step 1. First, we show that $C_{n}$ is closed and convex for all $n \in \mathbb{N}$.

Clearly $C_{1}=C$ is closed and convex. Suppose that $C_{n}$ is closed and convex for all $n \in \mathbb{N}$. For any $v \in C_{n}$, we know that $G\left(v, J z_{n}\right) \leq G\left(v, J x_{n}\right)+\zeta_{n}$ is equivalent to

$$
2\left\langle v, J x_{n}-J z_{n}\right\rangle \leq\left\|x_{n}\right\|^{2}-\left\|z_{n}\right\|^{2}+\zeta_{n}
$$

So, $C_{n+1}$ is closed and convex. Hence by induction $C_{n}$ is closed and convex for all $n \geq 1$.

Step 2. We will show that the sequence $\left\{x_{n}\right\}$ is well defined.

We will show by induction that $₹ \subset C_{n}$ for all $n \in \mathbb{N}$. It is obvious that $₹ \subset C_{1}=C$.

Suppose that $\mathcal{F} \subset C_{n}$ for some $n \in \mathbb{N}$. Let $q \in \mathcal{F}$, put $u_{n}=K_{n}^{m} x_{n}, K_{n}^{j}=T_{r_{j, n}}^{\theta_{j}} T_{r_{j-1, n}}^{\theta_{j-1}} \ldots T_{r_{1, n}}^{\theta_{1}}$ for all $j=1,2,3, \ldots, m, K_{n}^{0}=I$, we have that

$$
G\left(q, J u_{n}\right)=\& G\left(q, J K_{n}^{m} x_{n}\right) \leq \& G\left(q, J x_{n}\right)
$$

From (3.3) and $S$ which is a totally quasi- $\phi$ asymptotically nonexpansive mappings, it follows that

$$
\begin{aligned}
G\left(q, J z_{n}\right)= & G\left(q,\left(\alpha_{n} J x_{1}+\left(1-\alpha_{n}\right) J S^{n} u_{n}\right)\right) \\
= & \|q\|^{2}-2 \alpha_{n}\left\langle q, J x_{1}\right\rangle-2\left(1-\alpha_{n}\right)\left\langle q, J S^{n} u_{n}\right\rangle \\
& +\left\|\alpha_{n} J x_{1}+\left(1-\alpha_{n}\right) J S^{n} u_{n}\right\|^{2}+2 \rho f(q) \\
\leq & \|q\|^{2}-2 \alpha_{n}\left\langle q, J x_{1}\right\rangle-2\left(1-\alpha_{n}\right)\left\langle q, J S^{n} u_{n}\right\rangle \\
& +\alpha_{n}\left\|J x_{1}\right\|^{2}+\left(1-\alpha_{n}\right)\left\|J S^{n} u_{n}\right\|^{2}+2 \rho f(q) \\
= & \alpha_{n} G\left(q, J x_{1}\right)+\left(1-\alpha_{n}\right) G\left(q, J S^{n} u_{n}\right) \\
\leq & \alpha_{n} G\left(q, J x_{1}\right)+\left(1-\alpha_{n}\right)\left(G\left(q, J u_{n}\right)+v_{n} \psi\left(G\left(q, J u_{n}\right)\right)+\mu_{n}\right) \\
\leq & \alpha_{n} G\left(q, J x_{1}\right)+\left(1-\alpha_{n}\right) G\left(q, J x_{n}\right)+v_{n} \sup _{q \in \mathcal{F}} \psi\left(G\left(q, J x_{n}\right)\right)+\mu_{n} \\
= & \alpha_{n} G\left(q, J x_{1}\right)+\left(1-\alpha_{n}\right) G\left(q, J x_{n}\right)+\zeta_{n} .
\end{aligned}
$$

This shows that $q \in C_{n+1}$ which implies that $\mathcal{F} \subset C_{n+1}$, and hence, $\mathcal{F} \subset C_{n}$ for all $n \in \mathbb{N}$. and the sequence $\left\{x_{n}\right\}$ is well defined. From $x_{n}=\Pi_{C_{n}}^{f} x_{1}$, we see that

$$
\left\langle x_{n}-q, J x_{1}-J x_{n}\right\rangle+\rho f(q)-\rho f\left(x_{n}\right) \geq 0, \quad \forall q \in C_{n} .
$$

Since $\mathcal{F} \subset C_{n}$ for each $n \in \mathbb{N}$, we arrive at

$$
\left\langle x_{n}-q, J x_{1}-J x_{n}\right\rangle+\rho f(q)-\rho f\left(x_{n}\right) \geq 0, \quad \forall q \in \mathcal{F} .
$$

Hence, the sequence $\left\{x_{n}\right\}$ is well defined. 
Step 3. We will show that $x_{n} \rightarrow p \in \mathcal{F}:=F(S) \cap\left(\cap_{j=1}^{m} \operatorname{EP}\left(\theta_{j}\right)\right)$.

Let $f: E \rightarrow \mathbb{R}$ is convex and lower semicontinuous function, follows from Lemma 2.8, there exist $x^{*} \in E^{*}$ and $\alpha \in \mathbb{R}$ such that

$$
f(y) \geq\left\langle y, x^{*}\right\rangle+\alpha, \quad \forall y \in E
$$

Since $x_{n} \in C_{n} \subset E$, it follows that

$$
\begin{aligned}
G\left(x_{n}, J x_{1}\right) & =\left\|x_{n}\right\|^{2}-2\left\langle x_{n}, J x_{1}\right\rangle+\left\|x_{1}\right\|^{2}+2 \rho f\left(x_{n}\right) \\
& \geq\left\|x_{n}\right\|^{2}-2\left\langle x_{n}, J x_{1}\right\rangle+\left\|x_{1}\right\|^{2}+2 \rho\left\langle x_{n}, x^{*}\right\rangle+2 \rho \alpha \\
& =\left\|x_{n}\right\|^{2}-2\left\langle x_{n}, J x_{1}-\rho x^{*}\right\rangle+\left\|x_{1}\right\|^{2}+2 \rho \alpha \\
& \geq\left\|x_{n}\right\|^{2}-2\left\|x_{n}\right\|\left\|J x_{1}-\rho x^{*}\right\|+\left\|x_{1}\right\|^{2}+2 \rho \alpha \\
& =\left(\left\|x_{n}\right\|-\left\|J x_{1}-\rho x^{*}\right\|\right)^{2}+\left\|x_{1}\right\|^{2}-\left\|J x_{1}-\rho x^{*}\right\|^{2}+2 \rho \alpha .
\end{aligned}
$$

For $q \in \mathcal{F}$ and $x_{n}=\Pi_{C_{n}}^{f} x_{1}$, we have

$$
G\left(q, J x_{1}\right) \geq G\left(x_{n}, J x_{1}\right) \geq\left(\left\|x_{n}\right\|-\left\|J x_{1}-\rho x^{*}\right\|\right)^{2}+\left\|x_{1}\right\|^{2}-\left\|J x_{1}-\rho x^{*}\right\|^{2}+2 \rho \alpha .
$$

This shows that $\left\{x_{n}\right\}$ is bounded and so is $\left\{G\left(x_{n}, J x_{1}\right)\right\}$. From the fact that $x_{n+1}=\Pi_{C_{n+1}}^{f} x_{1} \in$ $C_{n+1} \subset C_{n}$ and $x_{n}=\Pi_{C_{n}}^{f} x_{1}$, it follows from Lemma 2.10 that

$$
0 \leq\left(\left\|x_{n+1}-\right\| x_{n} \|\right)^{2} \leq \phi\left(x_{n+1}, x_{n}\right) \leq G\left(x_{n+1}, J x_{1}\right)-G\left(x_{n}, J x_{1}\right) .
$$

That is, $\left\{G\left(x_{n}, J x_{1}\right)\right\}$ is nondecreasing. Hence, we obtain that $\lim _{n \rightarrow \infty} G\left(x_{n}, J x_{1}\right)$ exists. Taking $n \rightarrow \infty$, we obtain

$$
\lim _{n \rightarrow \infty} \phi\left(x_{n+1}, x_{n}\right)=0
$$

Since $E$ is reflexive, $\left\{x_{n}\right\}$ is bounded, and $C_{n}$ is closed and convex for all $n \in \mathbb{N}$. Without loss of generality, we can assume that $x_{n} \rightarrow p \in C_{n}$. From the fact that $x_{n}=\Pi_{C_{n}}^{f} x_{1}$, we get that

$$
G\left(x_{n}, J x_{1}\right) \leq G\left(p, J x_{1}\right), \quad \forall n \in \mathbb{N}
$$

Since $f$ is convex and lower semicontinuous, we have

$$
\begin{aligned}
\liminf _{n \rightarrow \infty} G\left(x_{n}, J x_{1}\right) & =\liminf _{n \rightarrow \infty}\left\{\left\|x_{n}\right\|^{2}-2\left\langle x_{n}, J x_{1}\right\rangle+\left\|x_{1}\right\|^{2}+2 \rho f\left(x_{n}\right)\right\} \\
& \geq\|p\|^{2}-2\left\langle p, J x_{1}\right\rangle+\left\|x_{1}\right\|^{2}+2 \rho f(p) \\
& =G\left(x_{n}, J x_{1}\right) .
\end{aligned}
$$


Journal of Applied Mathematics

By (3.12) and (3.13), we get

$$
G\left(p, J x_{1}\right) \leq \liminf _{n \rightarrow \infty} G\left(x_{n}, J x_{1}\right) \leq \limsup _{n \rightarrow \infty} G\left(x_{n}, J x_{1}\right) \leq G\left(p, J x_{1}\right) .
$$

That is, $\lim _{n \rightarrow \infty} G\left(x_{n}, J x_{1}\right)=G\left(p, J x_{1}\right)$; this implies that $\left\|x_{n}\right\| \rightarrow\|p\|$; by virtue of the KadecKlee property of $E$, we obtain that

$$
\lim _{n \rightarrow \infty} x_{n}=p
$$

We also have

$$
\lim _{n \rightarrow \infty} x_{n+1}=p
$$

From (3.15), we get that

$$
\lim _{n \rightarrow \infty} \zeta_{n}=\lim _{n \rightarrow \infty}\left(v_{n} \sup _{q \in \mathscr{F}} \psi\left(G\left(q, x_{n}\right)\right)+\mu_{n}\right)=0 .
$$

(a) We show that $p \in \cap_{j=1}^{m} \operatorname{EP}\left(\theta_{j}\right)$.

Since $x_{n+1}=\Pi_{C_{n+1}}^{f} x_{1} \in C_{n+1} \subset C_{n}$ and the definition of $C_{n+1}$, we have

$$
G\left(x_{n+1}, J u_{n}\right) \leq \alpha_{n} G\left(x_{n+1}, J x_{1}\right)+\left(1-\alpha_{n}\right) G\left(x_{n+1}, J x_{n}\right)+\zeta_{n}
$$

is equivalent to

$$
\phi\left(x_{n+1}, u_{n}\right) \leq \alpha_{n} \phi\left(x_{n+1}, x_{1}\right)+\left(1-\alpha_{n}\right) \phi\left(x_{n+1}, x_{n}\right)+\zeta_{n} .
$$

From (3.11), (3.15), and (3.17), it follows that

$$
\lim _{n \rightarrow \infty} \phi\left(x_{n+1}, u_{n}\right)=0
$$

From (2.7), we have

$$
\left(\left\|x_{n+1}\right\|-\left\|u_{n}\right\|\right)^{2} \longrightarrow 0
$$

Since $\left\|x_{n+1}\right\| \rightarrow\|p\|$, we have

$$
\left\|u_{n}\right\| \longrightarrow\|p\| \text { as } n \longrightarrow \infty
$$

It follow that

$$
\left\|J u_{n}\right\| \longrightarrow\|J p\| \quad \text { as } n \longrightarrow \infty \text {. }
$$


That is, $\left\{\left\|J u_{n}\right\|\right\}$ is bounded in $E^{*}$ and $E^{*}$ is reflexive; we assume that $J u_{n} \rightarrow u^{*} \in E^{*}$. In view of $J(E)=E^{*}$, there exists $u \in E$ such that $J u=u^{*}$. It follows that

$$
\begin{aligned}
\phi\left(x_{n+1}, u_{n}\right) & =\left\|x_{n+1}\right\|^{2}-2\left\langle x_{n+1}, J y_{n}\right\rangle+\left\|u_{n}\right\|^{2} \\
& =\left\|x_{n+1}\right\|^{2}-2\left\langle x_{n+1}, J u_{n}\right\rangle+\left\|J u_{n}\right\|^{2} .
\end{aligned}
$$

Taking $\liminf \operatorname{in}_{n \rightarrow \infty}$ on both sides of the equality above and $\|\cdot\|$ is the weak lower semicontinuous, it yields that

$$
\begin{aligned}
0 & \geq\|p\|^{2}-2\left\langle p, u^{*}\right\rangle+\left\|u^{*}\right\|^{2} \\
& =\|p\|^{2}-2\langle p, J u\rangle+\|J u\|^{2} \\
& =\|p\|^{2}-2\langle p, J u\rangle+\|u\|^{2} \\
& =\phi(p, u)
\end{aligned}
$$

That is, $p=u$, which implies that $u^{*}=J p$. It follows that $J u_{n} \rightarrow J p \in E^{*}$. From (3.23) and the Kadec-Klee property of $E^{*}$ we have $J u_{n} \rightarrow J p$ as $n \rightarrow \infty$. Note that $J^{-1}: E^{*} \rightarrow E$ is norm-weak *-continuous; that is, $u_{n} \rightarrow p$. From (3.22) and the Kadec-Klee property of $E$, we have

$$
\lim _{n \rightarrow \infty} u_{n}=p
$$

For $q \in F \subset C_{n}$, by nonexpansiveness, we observe that

$$
\begin{aligned}
\phi\left(q, u_{n}\right) & =\phi\left(q, K_{n}^{m} x_{n}\right) \\
& \leq \phi\left(q, K_{n}^{m-1} x_{n}\right) \\
& \leq \phi\left(q, K_{n}^{m-2} x_{n}\right) \\
& \vdots \\
& \leq \phi\left(q, K_{n}^{j} x_{n}\right) .
\end{aligned}
$$

By Lemma 2.14, we have for $j=1,2,3, \ldots, m$

$$
\phi\left(K_{n}^{j} x_{n}, x_{n}\right) \& \leq \phi\left(q, x_{n}\right)-\phi\left(q, K_{n}^{j} x_{n}\right) \leq \phi\left(q, x_{n}\right)-\phi\left(q, u_{n}\right) .
$$

Since $x_{n}, u_{n} \rightarrow p$ as $n \rightarrow \infty$, we get $\phi\left(K_{n}^{j} x_{n}, x_{n}\right) \rightarrow 0$ as $n \rightarrow \infty$, for $j=1,2,3, \ldots, m$. From (2.7), it follow that

$$
\left(\left\|K_{n}^{j} x_{n}\right\|-\left\|x_{n}\right\|\right)^{2} \longrightarrow 0
$$


Since $\left\|x_{n}\right\| \rightarrow\|p\|$, we also have

$$
\left\|K_{n}^{j} x_{n}\right\| \longrightarrow\|p\| \quad \text { as } n \longrightarrow \infty \text {. }
$$

Since $\left\{K_{n}^{j} x_{n}\right\}$ is bounded and $E$ is reflexive, without loss of generality we assume that $K_{n}^{j} y_{n} \rightarrow$ $h$. We know that $C_{n}$ is closed and convex for each $n \geq 1$ it is obvious that $h \in C_{n}$. Again since

$$
\phi\left(K_{n}^{j} x_{n}, x_{n}\right)=\left\|K_{n}^{j} x_{n}\right\|^{2}-2\left\langle K_{n}^{j} x_{n}, J x_{n}\right\rangle+\left\|x_{n}\right\|^{2},
$$

taking $\lim \inf _{n \rightarrow \infty}$ on the both sides of equality above, we have

$$
0 \& \geq\|h\|^{2}-2\langle h, J p\rangle+\|p\|^{2}=\phi(h, p) .
$$

That is, $h=p$, for all $j=1,2,3, \ldots, m$; it follow that

$$
K_{n}^{j} x_{n} \rightarrow p ;
$$

from (3.30), (3.33), and the Kadec-Klee property, it follows that

$$
\lim _{n \rightarrow \infty} K_{n}^{j} x_{n}=p, \quad \forall j=1,2,3, \ldots, m .
$$

By using triangle inequality, we have

$$
\left\|x_{n}-K_{n}^{j} x_{n}\right\| \leq\left\|x_{n}-p\right\|+\left\|p-K_{n}^{j} u_{n}\right\| .
$$

Since $x_{n}, K_{n}^{j} x_{n} \rightarrow p$ as $n \rightarrow \infty$, we have

$$
\lim _{n \rightarrow \infty}\left\|x_{n}-K_{n}^{j} x_{n}\right\|=0, \quad \forall j=1,2,3, \ldots, m .
$$

Again by using triangle inequality, we have

$$
\left\|K_{n}^{j} x_{n}-K_{n}^{j-1} x_{n}\right\| \leq\left\|K_{n}^{j} x_{n}-x_{n}\right\|+\left\|x_{n}-K_{n}^{j-1} x_{n}\right\| .
$$

From (3.36), we also have

$$
\lim _{n \rightarrow \infty}\left\|K_{n}^{j} x_{n}-K_{n}^{j-1} x_{n}\right\|=0, \quad \forall j=1,2,3, \ldots, m .
$$

Since $J$ is uniformly norm-to-norm continuous, we obtain

$$
\lim _{n \rightarrow \infty}\left\|J K_{n}^{j} x_{n}-J K_{n}^{j-1} x_{n}\right\|=0, \quad \forall j=1,2,3, \ldots, m .
$$


From $r_{j, n}>0$, we have $\left\|J K_{n}^{j} x_{n}-J K_{n}^{j-1} x_{n}\right\| / r_{j, n} \rightarrow 0$ as $n \rightarrow \infty$ for all $j=1,2,3, \ldots, m$, and

$$
\theta_{j}\left(K_{n}^{j} y_{n}, y\right)+\frac{1}{r_{j, n}}\left\langle y-K_{n}^{j} x_{n}, J K_{n}^{j} x_{n}-J K_{n}^{j-1} x_{n}\right\rangle \geq 0, \quad \forall y \in C
$$

By (A2), that

$$
\begin{aligned}
\left\|y-K_{n}^{j} y_{n}\right\| \frac{\left\|J K_{n}^{j} y_{n}-J K_{n}^{j-1} x_{n}\right\|}{r_{n}} & \geq \frac{1}{r_{j, n}}\left\langle y-K_{n}^{j} x_{n}, J K_{n}^{j} y_{n}-J K_{n}^{j-1} x_{n}\right\rangle \\
& \geq-\theta_{j}\left(K_{n}^{j} x_{n}, y\right) \\
& \geq \theta_{j}\left(y, K_{n}^{j} x_{n}\right), \quad \forall y \in C,
\end{aligned}
$$

and $K_{n}^{j} x_{n} \rightarrow p$ as $n \rightarrow \infty$, we get $\theta_{j}(y, p) \leq 0$, for all $y \in C$. For $0<t<1$, define $y_{t}=$ $t y+(1-t) p$, then $y_{t} \in C$ which imply that $\theta_{j}\left(y_{t}, p\right) \leq 0$. From (A1), we obtain that

$$
0=\theta_{j}\left(y_{t}, y_{t}\right) \leq t \theta_{j}\left(y_{t}, y\right)+(1-t) \theta_{j}\left(y_{t}, p\right) \leq t \theta_{j}\left(y_{t}, y\right)
$$

We have that $\theta_{j}\left(y_{t}, y\right) \geq 0$. From (A3), we have $\theta_{j}(p, y) \geq 0$, for all $y \in C$ and $j=1,2,3, \ldots, m$. That is, $p \in \operatorname{EP}\left(\theta_{j}\right)$, for all $j=1,2,3, \ldots, m$. This imply that $p \in \cap_{j=1}^{m} \operatorname{EP}\left(\theta_{j}\right)$.

(b) We show that $p \in F(S)$.

Since $x_{n+1}=\prod_{C_{n+1}}^{f} x_{1} \in C_{n+1} \subset C_{n}$ and the definition of $C_{n+1}$, we have

$$
G\left(x_{n+1}, J z_{n}\right) \leq \alpha_{n} G\left(x_{n+1}, J x_{1}\right)+\left(1-\alpha_{n}\right) G\left(x_{n+1}, J x_{n}\right)+\zeta_{n}
$$

is equivalent to

$$
\phi\left(x_{n+1}, z_{n}\right) \leq \alpha_{n} \phi\left(x_{n+1}, x_{1}\right)+\left(1-\alpha_{n}\right) \phi\left(x_{n+1}, x_{n}\right)+\zeta_{n} .
$$

Following (3.11), (3.15), and (3.17), we get that

$$
\lim _{n \rightarrow \infty} \phi\left(x_{n+1}, z_{n}\right)=0 .
$$

From (2.7), we also have

$$
\left\|z_{n}\right\| \longrightarrow\|p\| \quad \text { as } n \longrightarrow \infty \text {. }
$$

It follows that

$$
\left\|J z_{n}\right\| \longrightarrow\|J p\| \text { as } n \longrightarrow \infty \text {. }
$$


This implies that $\left\{\left\|J z_{n}\right\|\right\}$ is bounded in $E^{*}$. Since $E$ is reflexive and $E^{*}$ is also reflexive, we can assume that $J z_{n} \rightarrow z^{*} \in E^{*}$. In view of the reflexive of $E$, we see that $J(E)=E^{*}$. There exists $z \in E$ such that $J z=z^{*}$. It follows that

$$
\begin{aligned}
\phi\left(x_{n+1}, z_{n}\right) & =\left\|x_{n+1}\right\|^{2}-2\left\langle x_{n+1}, J z_{n}\right\rangle+\left\|z_{n}\right\|^{2} \\
& =\left\|x_{n+1}\right\|^{2}-2\left\langle x_{n+1}, J z_{n}\right\rangle+\left\|J z_{n}\right\|^{2} .
\end{aligned}
$$

Taking $\lim \inf _{n \rightarrow \infty}$ on both sides of the equality above and in view of the weak lower semicontinuity of norm $\|\cdot\|$, it yields that

$$
\begin{aligned}
0 & \geq\|p\|^{2}-2\left\langle p, z^{*}\right\rangle+\left\|z^{*}\right\|^{2} \\
& =\|p\|^{2}-2\langle p, J z\rangle+\|J z\|^{2} \\
& =\|p\|^{2}-2\langle p, J z\rangle+\|z\|^{2} \\
& =\phi(p, z)
\end{aligned}
$$

That is $p=z$, which implies that $z^{*}=J p$. It follows that $J z_{n} \rightarrow J p \in E^{*}$.From (3.47) and the Kadec-Klee property of $E^{*}$ we have $J z_{n} \rightarrow J p$ as $n \rightarrow \infty$. Since $J^{-1}: E^{*} \rightarrow E$ is normweak ${ }^{*}$-continuous, $z_{n} \rightarrow p$ as $n \rightarrow \infty$. From (3.46) and the Kadec-Klee property of $E$, we have

$$
\lim _{n \rightarrow \infty} z_{n}=p
$$

Since $\left\{x_{n}\right\}$ is bounded, then a mapping $S$ is also bounded. From the condition $\lim _{n \rightarrow \infty} \alpha_{n}=0$, we have that

$$
\left\|J z_{n}-J S^{n} u_{n}\right\|=\lim _{n \rightarrow \infty} \alpha_{n}\left\|J x_{1}-J S^{n} u_{n}\right\|=0
$$

From (3.47), we get

$$
\left\|J S^{n} u_{n}\right\| \longrightarrow\|J p\| \quad \text { as } n \longrightarrow \infty
$$

Since $J^{-1}: E^{*} \rightarrow E$ is norm-weak*-continuous,

$$
S^{n} u_{n} \rightarrow p \quad \text { as } n \longrightarrow \infty
$$

On the other hand, we observe that

$$
\left|\left\|S^{n} u_{n}\right\|-\|p\|\right|=\left\|J\left(S^{n} u_{n}\right)\right\|-\|J p\| \leq\left\|J\left(S^{n} u_{n}\right)-J p\right\| .
$$

In view of (3.52), we obtain $\left\|S^{n} u_{n}\right\| \rightarrow\|p\|$. Since $E$ has the Kadee-Klee property, we get

$$
S^{n} u_{n} \longrightarrow p \text { for each } n \in \mathbb{N} \text {. }
$$


From $S^{n} u_{n} \rightarrow p$, we get $S^{n+1} u_{n} \rightarrow p$; that is, $S S^{n} u_{n} \rightarrow p$. In view of closeness of $S$, we have $S p=p$. This implies that $p \in F(S)$. From (a) and (b), it follows that $p \in \cap_{j=1}^{m} \operatorname{EP}\left(\theta_{j}\right) \cap F(S)$.

Step 4. We will show that $p=\Pi_{\mathscr{q}}^{f} x_{1}$.

Since $\mathcal{F}$ is closed and convex set from Lemma 2.9, we have $\Pi_{\mathcal{F}}^{f} x_{1}$ which is single valued, denoted by $v$. By definition $x_{n}=\Pi_{C_{n}}^{f} x_{1}$ and $v \in \mathcal{F} \subset C_{n}$, we also have

$$
G\left(x_{n}, J x_{1}\right) \leq \mathrm{G}\left(v, J x_{1}\right), \quad \forall n \geq 1 .
$$

By the definition of $G$ and $f$, we know that, for each given $x, G(\xi, J x)$ is convex and lower semicontinuous with respect to $\xi$. So

$$
G\left(p, J x_{1}\right) \leq \liminf _{n \rightarrow \infty} G\left(x_{n}, J x_{1}\right) \leq \limsup _{n \rightarrow \infty} G\left(x_{n}, J x_{1}\right) \leq G\left(v, J x_{1}\right) .
$$

From the definition of $\Pi_{\mathscr{F}}^{f} x_{1}$ and since $p \in \mathcal{F}$, we conclude that $v=p=\Pi_{\mathscr{F}}^{f} x_{1}$ and $x_{n} \rightarrow p$ as $n \rightarrow \infty$. The proof is completed.

Setting $v_{n} \equiv 0$ and $\mu_{n} \equiv 0$ in Theorem 3.1, then we have the following corollary.

Corollary 3.2. Let $C$ be a nonempty, closed, and convex subset of a uniformly smooth and strictly convex Banach space $E$ with the Kadec-Klee property. For each $j=1,2, \ldots, m$, let $\theta_{j}$ be a bifunction from $C \times C$ to $\mathbb{R}$ which satisfies conditions (A1)-(A4). Let $S: C \rightarrow C$ be a closed and quasi- $\phi-$ asymptotically nonexpansive mappings, and let $f: E \rightarrow \mathbb{R}$ be a convex and lower semicontinuous function with $C \subset$ int $(D(f))$ such that $f(x) \geq 0$ for all $x \in C$ and $f(0)=0$. Assume that $\mathcal{F}=F(S) \cap\left(\cap_{j=1}^{m} \mathrm{EP}\left(\theta_{j}\right)\right) \neq \emptyset$. For an initial point $x_{1} \in E$ and $C_{1}=C$, we define the sequence $\left\{x_{n}\right\}$ by

$$
\begin{gathered}
u_{n}=T_{r_{m, n}}^{\theta_{m}} T_{r_{m-1, n}}^{\theta_{m-1}} T_{r_{m-2, n}}^{\theta_{m-2}} \cdots T_{r_{1, n}}^{\theta_{1}} x_{n} \\
z_{n}=J^{-1}\left(\alpha_{n} J x_{1}+\left(1-\alpha_{n}\right) J S^{n} u_{n}\right), \\
C_{n+1}=\left\{v \in C_{n}: G\left(v, J z_{n}\right) \leq G\left(v, J u_{n}\right) \leq G\left(v, J x_{1}\right)+\left(1-\alpha_{n}\right) G\left(v, J x_{n}\right)+\zeta_{n}\right\} \\
x_{n+1}=\prod_{C_{n+1}}^{f} x_{1}, \quad n \in \mathbb{N},
\end{gathered}
$$

where $\left\{\alpha_{n}\right\}$ is a sequence in $[0,1], \zeta_{n}=v_{n} \sup _{q \in \mathcal{F}} \psi\left(G\left(q, x_{n}\right)\right)+\mu_{n}$, and $\left\{r_{j, n}\right\} \subset[d, \infty)$ for some $d>0$. If $\lim _{n \rightarrow \infty} \alpha_{n}=0$, then $\left\{x_{n}\right\}$ converges strongly to $\Pi_{\mp}^{f} x_{1}$.

Let $E$ be a real Banach space, and let $C$ be a nonempty closed convex subset of $E$. Given a mapping $A: C \rightarrow E^{*}$, let $\theta(x, y)=\langle A x, y-x\rangle$ for all $x, y \in C$. Then $x^{*} \in \operatorname{EP}(\theta)$ if and only if $\left\langle A x^{*}, y-x^{*}\right\rangle \geq 0$ for all $y \in C$; that is, $x^{*}$ is a solution of the classical variational inequality problem. The set of this solution is denoted by $\operatorname{VI}(A, C)$. For each $r>0$ and $x \in E$, we define 
the mapping $T_{r}^{\theta} x$ by

$$
T_{r}^{\theta} x=\left\{z \in C:\langle A z, y-z\rangle+\frac{1}{r}\langle y-z, J z-J x\rangle \geq 0, \forall y \in C\right\} .
$$

Hence, we obtain the following corollary.

Corollary 3.3. Let $C$ be a nonempty, closed, and convex subset of a uniformly smooth and strictly convex Banach space $E$ with the Kadec-Klee property. For each $j=1,2, \ldots, m$, let $\left\{A_{j}\right\}$ be a continuous monotone mapping of $C$ into $E^{*}$. Let $S: C \rightarrow C$ be a closed totally quasi- $\phi$-asymptotically nonexpansive mappings with nonnegative real sequences $v_{n}, \mu_{n}$ with $v_{n} \rightarrow 0, \mu_{n} \rightarrow 0$ as $n \rightarrow \infty$ and a strictly increasing continuous function $\psi: \mathbb{R}^{+} \rightarrow \mathbb{R}^{+}$with $\psi(0)=0$, and let $f: E \rightarrow \mathbb{R}$ be a convex and lower semicontinuous function with $C \subset$ int $(D(f))$ such that $f(x) \geq 0$ for all $x \in C$ and $f(0)=0$. Assume that $\mathcal{F}=F(S) \cap\left(\cap_{j=1}^{m} \operatorname{VI}\left(\mathrm{A}_{j}, \mathrm{C}\right)\right) \neq \emptyset$. For an initial point $x_{1} \in E$ and $C_{1}=C$, one defines the sequence $\left\{x_{n}\right\}$ by

$$
\begin{gathered}
u_{n}=T_{r_{m, n}}^{\theta_{m}} T_{r_{m-1, n}}^{\theta_{m-1}} T_{r_{m-2, n}}^{\theta_{m-2}} \cdots T_{r_{1, n}}^{\theta_{1}} x_{n} \\
z_{n}=J^{-1}\left(\alpha_{n} J x_{1}+\left(1-\alpha_{n}\right) J S^{n} u_{n}\right), \\
C_{n+1}=\left\{v \in C_{n}: G\left(v, J z_{n}\right) \leq G\left(v, J u_{n}\right) \leq G\left(v, J x_{1}\right)+\left(1-\alpha_{n}\right) G\left(v, J x_{n}\right)+\zeta_{n}\right\} \\
x_{n+1}=\Pi_{C_{n+1}}^{f} x_{1}, \quad n \in \mathbb{N},
\end{gathered}
$$

where $\zeta_{n}=v_{n} \sup _{q \in \mathcal{F}} \psi\left(G\left(q, x_{n}\right)\right)+\mu_{n},\left\{\alpha_{n}\right\}$ is a sequence in $[0,1]$, and $\left\{r_{j, n}\right\} \subset[d, \infty)$ for some $d>0$. If $\lim _{n \rightarrow \infty} \alpha_{n}=0$, then $\left\{x_{n}\right\}$ converges strongly to $\Pi_{\leftarrow}^{f} x_{1}$.

If $f(x)=0$ for all $x \in E$, we have $G(\xi, J x)=\phi(\xi, x)$ and $\Pi_{C}^{f} x=\Pi_{C} x$. From Theorem 3.1, we obtain the following corollary.

Corollary 3.4. Let $C$ be a nonempty, closed, and convex subset of a uniformly smooth and strictly convex Banach space $E$ with the Kadec-Klee property. For each $j=1,2, \ldots, m$, let $\theta_{j}$ be a bifunction from $C \times C$ to $\mathbb{R}$ which satisfies conditions (A1)-(A4). Let $S: C \rightarrow C$ be a closed totally quasi- $\phi$ asymptotically nonexpansive mappings with nonnegative real sequences $v_{n}, \mu_{n}$ with $v_{n} \rightarrow 0, \mu_{n} \rightarrow 0$ as $n \rightarrow \infty$ and a strictly increasing continuous function $\psi: \mathbb{R}^{+} \rightarrow \mathbb{R}^{+}$with $\psi(0)=0$. Assume that $\mathcal{F}=F(S) \cap\left(\cap_{j=1}^{m} \mathrm{EP}\left(\theta_{j}\right)\right) \neq \emptyset$. For an initial point $x_{1} \in E$ and $C_{1}=C$, we define the sequence $\left\{x_{n}\right\}$ by

$$
\begin{gathered}
u_{n}=T_{r_{m, n}}^{\theta_{m}} T_{r_{m-1, n}}^{\theta_{m-1}} T_{r_{m-2, n}}^{\theta_{m-2}} \cdots T_{r_{1, n}}^{\theta_{1}} x_{n} \\
z_{n}=J^{-1}\left(\alpha_{n} J x_{1}+\left(1-\alpha_{n}\right) J S^{n} u_{n}\right), \\
C_{n+1}=\left\{v \in C_{n}: G\left(v, J z_{n}\right) \leq G\left(v, J u_{n}\right) \leq G\left(v, J x_{1}\right)+\left(1-\alpha_{n}\right) G\left(v, J x_{n}\right)+\zeta_{n}\right\}, \\
x_{n+1}=\prod_{C_{n+1}} x_{1}, \quad n \in \mathbb{N},
\end{gathered}
$$

where $\left\{\alpha_{n}\right\}$ is a sequence in $[0,1], \zeta_{n}=v_{n} \sup _{q \in \mathcal{F}} \psi\left(G\left(q, x_{n}\right)\right)+\mu_{n}$, and $\left\{r_{j, n}\right\} \subset[d, \infty)$ for some $d>0$. If $\lim _{n \rightarrow \infty} \alpha_{n}=0$, then $\left\{x_{n}\right\}$ converges strongly to $\Pi_{\mp} x_{1}$. 
Remark 3.5. Our main result extends and improves the result of Chang et al. [13] in the following sense.

(i) From the algorithm we used new method replace by the generalized $f$-projection method which is more general than generalized projection.

(ii) For the problem, we extend the result to a common problem of fixed point problems and equilibrium problems.

\section{Acknowledgments}

The authors would like to thank The National Research Council of Thailand (NRCT) and Faculty of Science, King Mongkut's University of Technology Thonburi (Grant NRCT-2555). Furthermore, the authors would like to express their thanks to the referees for their helpful comments.

\section{References}

[1] W. R. Mann, "Mean value methods in iteration," Proceedings of the American Mathematical Society, vol. 4, pp. 506-510, 1953.

[2] H. H. Bauschke, E. Matoušková, and S. Reich, "Projection and proximal point methods: convergence results and counterexamples," Nonlinear Analysis. Theory, Methods E Applications, vol. 56, no. 5, pp. 715-738, 2004.

[3] A. Genel and J. Lindenstrauss, "An example concerning fixed points," Israel Journal of Mathematics, vol. 22, no. 1, pp. 81-86, 1975.

[4] B. Halpern, "Fixed points of nonexpanding maps," Bulletin of the American Mathematical Society, vol. 73, pp. 957-961, 1967.

[5] X. L. Qin, Y. J. Cho, S. M. Kang, and H. Y. Zhou, "Convergence of a modified Halpern-type iteration algorithm for quasi- $\phi$-nonexpansive mappings," Applied Mathematics Letters, vol. 22, no. 7, pp. 10511055, 2009.

[6] Z. M. Wang, Y. F. Su, D. X. Wang, and Y. C. Dong, “A modified Halpern-type iteration algorithm for a family of hemi-relatively nonexpansive mappings and systems of equilibrium problems in Banach spaces," Journal of Computational and Applied Mathematics, vol. 235, no. 8, pp. 2364-2371, 2011.

[7] W. Takahashi and K. Zembayashi, "Strong convergence theorem by a new hybrid method for equilibrium problems and relatively nonexpansive mappings," Fixed Point Theory and Applications, vol. 2008, Article ID 528476, 11 pages, 2008.

[8] W. Takahashi and K. Zembayashi, "Strong and weak convergence theorems for equilibrium problems and relatively nonexpansive mappings in Banach spaces," Nonlinear Analysis, vol. 70, no. 1, pp. 45-57, 2009.

[9] X. Li, N. Huang, and D. O’Regan, "Strong convergence theorems for relatively nonexpansive mappings in Banach spaces with applications," Computers $\mathcal{E}$ Mathematics with Applications, vol. 60, no. 5, pp. 1322-1331, 2010.

[10] E. U. Ofoedu and Y. Shehu, "Convergence analysis for finite family of relatively quasi nonexpansive mappings and systems of equilibrium problems," Applied Mathematics and Computation, vol. 217, no. 22, pp. 9142-9150, 2011.

[11] S.-S. Chang, H. W. J. Lee, and C. K. Chan, "A new hybrid method for solving a generalized equilibrium problem, solving a variational inequality problem and obtaining common fixed points in Banach spaces, with applications," Nonlinear Analysis. Theory, Methods E Applications, vol. 73, no. 7, pp. 2260-2270, 2010.

[12] X. Qin and Y. Su, "Strong convergence theorems for relatively nonexpansive mappings in a Banach space," Nonlinear Analysis. Theory, Methods E Applications, vol. 67, no. 6, pp. 1958-1965, 2007.

[13] S.-S. Chang, H. W. Joseph Lee, C. K. Chan, and L. Yang, "Approximation theorems for total quasi- $\phi-$ asymptotically nonexpansive mappings with applications," Applied Mathematics and Computation, vol. 218, no. 11, pp. 6489-6497, 2012. 
[14] X. Qin, Y. J. Cho, and S. M. Kang, "Convergence theorems of common elements for equilibrium problems and fixed point problems in Banach spaces," Journal of Computational and Applied Mathematics, vol. 225, no. 1, pp. 20-30, 2009.

[15] H. Zhou, G. Gao, and B. Tan, "Convergence theorems of a modified hybrid algorithm for a family of quasi- $\phi$-asymptotically nonexpansive mappings," Journal of Applied Mathematics and Computing, vol. 32, no. 2, pp. 453-464, 2010.

[16] Y. I. Alber, "Metric and generalized projection operators in Banach spaces: properties and applications," in Theory and Applications of Nonlinear Operators of Accretive and Monotone Type, vol. 178, pp. 15-50, Dekker, New York, NY, USA, 1996.

[17] Ya. I. Alcprimeber and S. Reich, "An iterative method for solving a class of nonlinear operator equations in Banach spaces," Panamerican Mathematical Journal, vol. 4, no. 2, pp. 39-54, 1994.

[18] I. Cioranescu, Geometry of Banach Spaces, Duality Mappings and Nonlinear Problems, vol. 62, Kluwer, Dordrecht, Germany, 1990.

[19] S. Kamimura and W. Takahashi, "Strong convergence of a proximal-type algorithm in a Banach space," SIAM Journal on Optimization, vol. 13, no. 3, pp. 938-945, 2002.

[20] W. Takahashi, Nonlinear Functional Analysis, Yokohama, 2000.

[21] K. Q. Wu and N. J. Huang, "The generalized $f$-projection operator with an application," Bulletin of the Australian Mathematical Society, vol. 73, no. 2, pp. 307-317, 2006.

[22] K. Goebel and S. Reich, Uniform Convexity, Hyperbolic Geometry, and Nonexpansive Mappings, vol. 83, Marcel Dekker, New York, NY, USA, 1984.

[23] S. Reich, "Book review: geometry of banach spaces, duality mappings and nonlinear problems," American Mathematical Society, vol. 26, no. 2, pp. 367-370, 1992.

[24] H. Hudzik, W. Kowalewski, and G. Lewicki, "Approximate compactness and full rotundity in Musielak-Orlicz spaces and Lorentz-Orlicz spaces," Zeitschrift für Analysis und ihre Anwendungen, vol. 25, no. 2, pp. 163-192, 2006.

[25] S.-S. Chang, H. W. Joseph Lee, C. K. Chan, and W. B. Zhang, "Approximation theorems for total quasi$\phi$-asymptotically nonexpansive mappings with applications," Applied Mathematics and Computation, vol. 218, no. 6, pp. 2921-2931, 2011.

[26] J. L. Fan, X. Liu, and J. Li, "Iterative schemes for approximating solutions of generalized variational inequalities in Banach spaces," Nonlinear Analysis. Theory, Methods E Applications, vol. 70, no. 11, pp. 3997-4007, 2009.

[27] K. Deimling, Nonlinear Functional Analysis, Springer, Berlin, Germany, 1985.

[28] E. Blum and W. Oettli, "From optimization and variational inequalities to equilibrium problems," The Mathematics Student, vol. 63, no. 1-4, pp. 123-145, 1994.

[29] F. Kohsaka and W. Takahashi, "Existence and approximation of fixed points of firmly nonexpansivetype mappings in Banach spaces," SIAM Journal on Optimization, vol. 19, no. 2, pp. 824-835, 2008. 


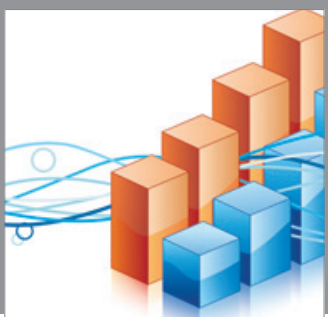

Advances in

Operations Research



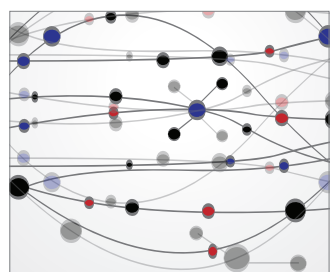

\section{The Scientific} World Journal
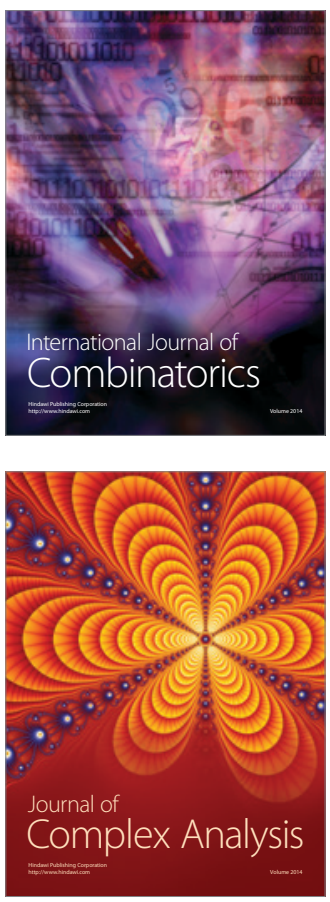

International Journal of

Mathematics and

Mathematical

Sciences
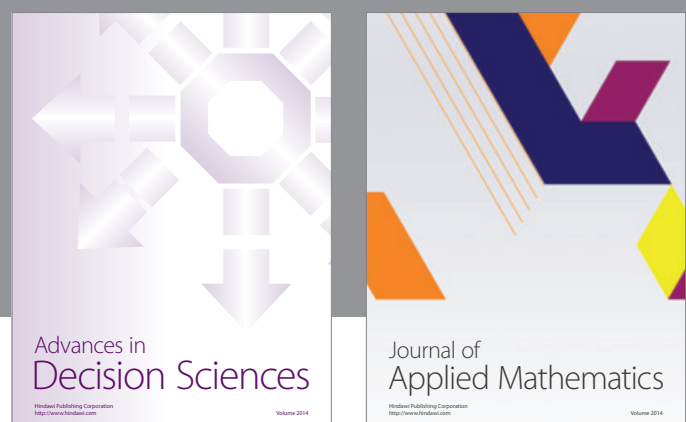

Journal of

Applied Mathematics
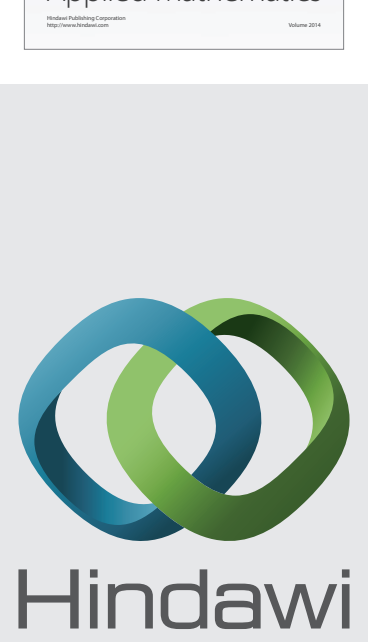

Submit your manuscripts at http://www.hindawi.com
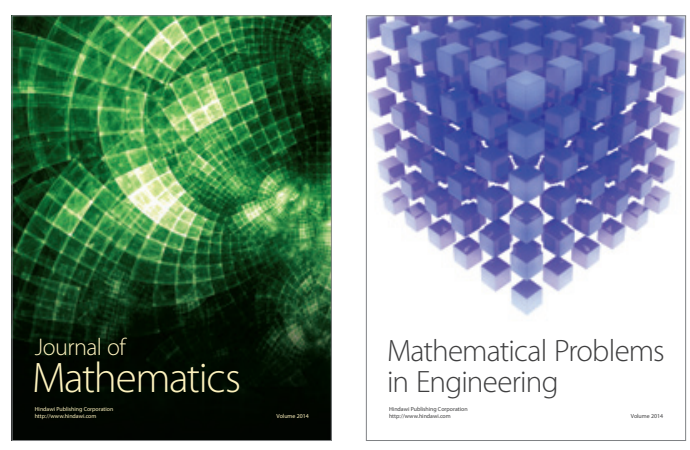

Mathematical Problems in Engineering
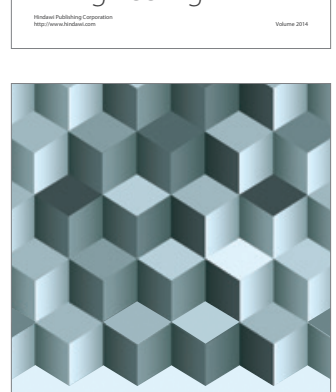

Journal of

Function Spaces
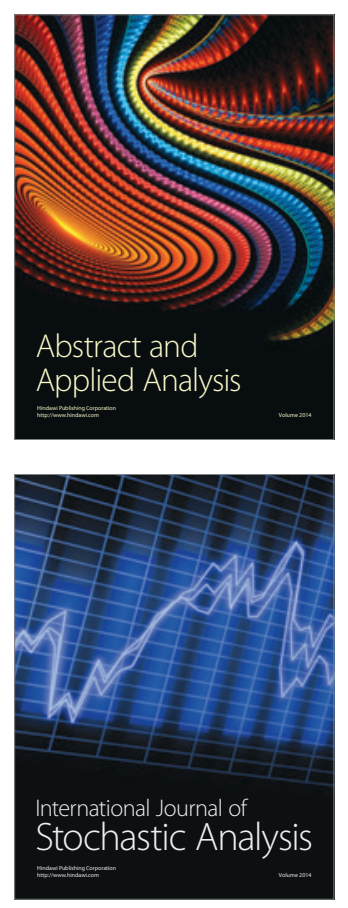

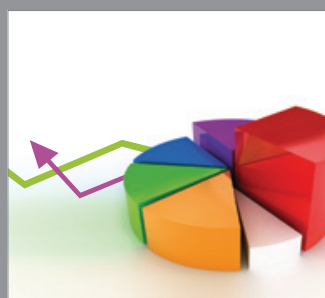

ournal of

Probability and Statistics

Promensencen
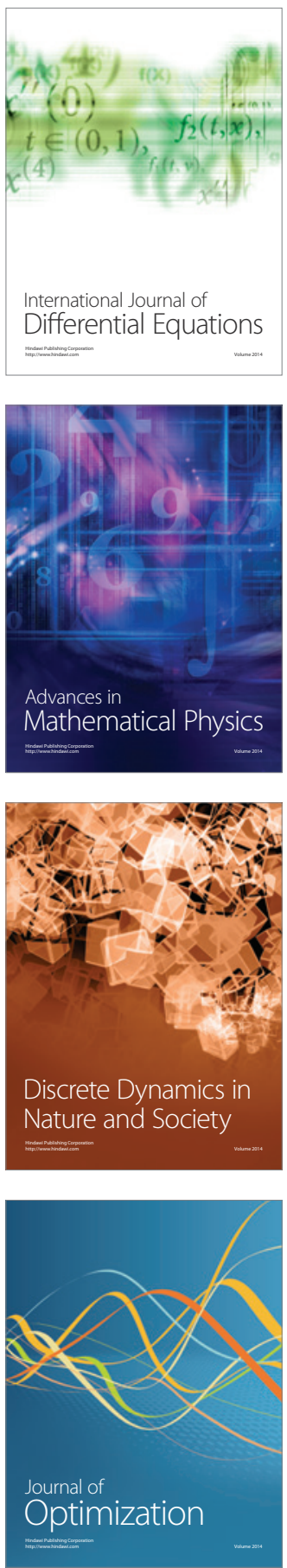